\title{
251.
}

\section{SUR QUELQUES FORMULES POUR LA DIFFÉRENTIATION.}

[From the Annali di Matematica pura ed applicata (Tortolini) tom. II. (1859), pp. 214-230. Traduction par l'auteur d'un mémoire présenté à la Société Royale de Londres le 26 Novembre, 1857.]

EN cherchant une formule dans la théorie des intégrales définies multiples je fus conduit il y a plusieurs années à chercher les coefficients différentiels successifs de l'expression $(\sqrt{x+\lambda}-\sqrt{x+\mu})^{2 i}$, et les résultats que j'ai trouvés sont donnés dans le Mémoire "On certain formulæ for differentiation with applications to the evaluation of definite integrals," Camb. and Dublin Mathematical Journal, tom. II. pp. 122-128 (1847), [41]. J'ai depuis cherché les coefficients différentiels successifs de l'expression plus générale $[(x+\lambda)(x+\mu)]^{3 k}(\sqrt{x+\lambda}-\sqrt{x+\mu})^{2 i}$, mais l'investigation n'était pas achevée. Mon attention fut rappelée à ce sujet par deux identités remarquables trouvées par le Prof. Donkin dans son Mémoire "On the equation of Laplace's functions etc." Phil. Trans. tom. CXLVII. (1857), pp. 43-57, au moyen de la comparaison de ses résultats avec ceux du Prof. Boole; identités qui appartenaient, je l'aperçus, à la classe des formules ci-dessus mentionnées: la première de ces deux identités se déduit en effet asseż facilement d'une formule exposée dans mon mémoire; la démonstration de la seconde identité est beaucoup plus difficile, et je n'ai réussi à l'établir qu'en la faisant dépendre de l'établissement de l'égalité des coefficients numériques de deux expressions de la même forme. Je suis depuis revenu aux investigations incomplètes dont j'ai parlé ci-dessus et les résultats que j’ai trouvés sont donnés dans le présent mémoire. Je remarque qu'en écrivant pour abréger

$$
P=2 x+\lambda+\mu, \quad Q=\sqrt{(x+\lambda)(x+\mu}), \quad R=(\sqrt{x+\lambda}-\sqrt{x+\mu})^{2},
$$

le sujet auquel appartiennent tous les résultats est la différentiation de l'expression $P^{a} Q^{\beta} R^{\gamma}$ : l'expression ci-dessus mentionnée $[(x+\lambda)(x+\mu)]^{1 k}(\sqrt{x+\lambda}-\sqrt{x+\mu})^{2 i}$ est de la 
forme dont il s'agit, et la question qui s'y rapporte est celle d'obtenir le développement de $D_{x}^{\mu} P^{\alpha} Q^{\beta} R^{\gamma}$, où $\alpha=0$.

La question suggérée par la seconde identité du Prof. Donkin est celle d'obtenir le développement de $\left(P^{-1}\left(Q^{4} D_{x}\right)^{\mu} P^{\alpha} Q^{\beta} R^{\gamma}\right.$, où $\alpha=\gamma-\beta$. Comme la démonstration de ces identités est l'un des objets de ce Mémoire, j'ai donné dans la première section la réduction des identités à la forme sous laquelle je les ai depuis considérées. La seconde section se rapporte au développement de l'expression $D_{x}^{\mu} P^{\alpha} Q^{\beta} R^{\gamma}$, où $\alpha=0$; la troisième section à celui de l'expression $\left(P^{-1} Q^{4} D_{x}\right)^{\mu} P^{\alpha} Q^{\beta} R^{\gamma}$, où $\alpha=\gamma-\beta$ : enfin la quatrième section contient l'application des deux identités, et quelques autres applications des formules.

\section{$\S \mathrm{I}$.}

1. La première des deux identités du Prof. Donkin est

$$
\left(\sin \theta D_{\theta} \sin \theta\right)^{n}\left(\operatorname{tang} \frac{1}{2} \theta\right)^{n}=1.3 .5 \ldots(2 n-1)(\sin \theta)^{2 n},
$$

laquelle est l'équation (27) article No. 14, de son Mémoire( $\left.{ }^{(}\right)$.

En écrivant $\cot \theta=t$, l'équation devient

$$
(-)^{n} D_{t}{ }^{n} \frac{\left(\sqrt{1+t^{2}}-t\right)^{n}}{\sqrt{1+t^{2}}}=\frac{1.3 .5 \ldots(2 n-1)}{\left(1+t^{2}\right)^{n+\frac{1}{2}}},
$$

et en posant comme à l'ordinaire $i=\sqrt{-1}$ on obtient

$$
\sqrt{1+t^{2}}-t=-\frac{1}{2}(\sqrt{t+i}-\sqrt{t-i})^{2}
$$

et en écrivant aussi

$$
1.3 .5 \ldots(2 n-1)=2^{n} \cdot \frac{1}{2} \cdot \frac{3}{2} \cdot \frac{5}{2} \ldots\left(n-\frac{1}{2}\right)=2^{n}\left[n-\frac{1}{2}\right]^{n},
$$

la formule devient

$$
D_{t}{ }^{n} \frac{(\sqrt{t+i}-\sqrt{t-i})^{2 n}}{\sqrt{1+t^{2}}}=\frac{2^{n}\left[n-\frac{1}{2}\right]^{n}}{\left(1+t^{2}\right)^{n+1}} .
$$

Ceci est un cas particulier de

$$
D_{x}{ }^{n} \frac{(\sqrt{x+\lambda}-\sqrt{x+\mu})^{2 n}}{\sqrt{(x+\lambda)(x+\mu)}}=\frac{(-)^{n}(R-\mu)^{2 n}\left[n-\frac{1}{2}\right]^{n}}{[(x+\lambda)(x+\mu)]^{n+1}},
$$

ou, en écrivant comme auparavant

$$
P=2 x+\lambda+\mu, \quad Q=\sqrt{(x+\lambda)(x+\mu)}, \quad R=[\sqrt{x+\lambda}-\sqrt{x+\mu}]^{2},
$$

la formule est

$$
D_{x}^{n} Q^{-1} R^{n}=(-)^{n}(\lambda-\mu)^{n n}\left[n-\frac{1}{2}\right] Q^{-2 n-1} .
$$

${ }^{1}$ Vedi la nota in fine. [This Note by the Editor, Professor Tortolini, relating merely to the transformation $\left(\sin \theta D_{\theta} \sin \theta\right)^{n}=(\sin \theta)^{-1}\left(\sin ^{2} \theta D_{\theta}\right)^{n} \sin \theta$, is not here reproduced.] 
2. (1) La comparaison mentionnée article No. 5 du Mémoire du Prof. Donkin donne $(\sin \theta)^{-n}\left(\sin \theta D_{\theta} \sin \theta\right)^{n}\left\{f\left[e^{\phi N-1} \operatorname{tang} \frac{1}{2} \theta+F\left(\frac{e^{\phi N-1}}{\operatorname{tang} \frac{1}{2} \theta}\right)\right]\right\}$

$$
\begin{aligned}
=\mu^{n}\left(D_{\mu} \frac{1}{\mu}\right)^{n}\{(\mu+ & \left.\mu^{2}\right)^{n} f\left(\frac{\mu}{1+\mu} \frac{\sqrt{1-\mu^{\prime 2}}}{\mu^{\prime}} e^{\phi N-1}\right) \\
& \left.+(-)^{n}\left(\mu-\mu^{2}\right)^{n} F\left(\frac{\mu}{1-\mu} \frac{\sqrt{1-\mu^{\prime 2}}}{\mu^{\prime}} e^{\phi N-1}\right)\right\},
\end{aligned}
$$

où $\mu=\cos \theta$, et après les différentiations $\mu^{\prime}=\cos \theta$. Les parties qui contiennent les fonctions indéterminées $f$ et $F$ doivent être égales, chacune prise à part de l'autre. Les parties qui contiennent $f$ seront égales, si cette égalité subsiste pour $f x=x^{\varsigma}$, où $s$ est un indice quelconque; c'est-à-dire l'égalité subsistera si

$(\sin \theta)^{-n}\left(\sin \theta D_{\theta} \sin \theta\right)^{n}\left(\operatorname{tang} \frac{1}{2} \theta\right)^{s}$

$$
\begin{aligned}
& =\mu^{n}\left(D_{\mu} \frac{1}{\mu}\right)^{n}\left(\mu+\mu^{2}\right)^{n} \frac{\mu^{8}}{(1+\mu)^{s}}\left(\frac{\sqrt{1-\mu^{\prime 2}}}{\mu^{\prime}}\right)^{s}, \\
& =\mu^{n}\left(\frac{\sqrt{1-\mu^{\prime 2}}}{\mu^{\prime}}\right)^{s}\left(D_{\mu} \frac{1}{\mu}\right)^{n}\left(\mu+\mu^{2}\right)^{n} \frac{\mu^{s}}{(1+\mu)^{s}},
\end{aligned}
$$

ou enfin en écrivant $\mu$, au lieu de $\mu^{\prime}$, si

$$
(\sin \theta)^{-n}\left(\sin \theta D_{\theta} \sin \theta\right)^{n}\left(\operatorname{tang} \frac{1}{2} \theta\right)^{s}=\mu^{n-s}\left(1-\mu^{2}\right)^{\frac{1}{s}}\left(D_{\mu} \frac{1}{\mu}\right)^{n} \mu^{n+8}(1+\mu)^{n-s},
$$

où $\mu=\cos \theta$; c'est en effet la seconde des deux identités du Prof. Donkin. L'égalité des parties qui contiennent $F^{\prime}$ dépend de la même manière de l'équation

$$
(\sin \theta)^{-n}\left(\sin \theta D_{\theta} \sin \theta\right)^{n}\left(\operatorname{tang} \frac{1}{2} \theta\right)^{s}=(-)^{n}\left(1-\mu^{2}\right)^{\frac{1 s}{s}}\left(D_{\mu} \frac{1}{\mu}\right)^{2} \mu^{n+s}(1-\mu)^{n-s},
$$

laquelle se déduit de l'autre équation en y écrivant $180^{\circ}-\theta$ au lieu de $\theta$.

3. La seconde identité (voir article No. 16) est

$$
(\sin \theta)^{-n}\left(\sin \theta D_{\theta} \sin \theta\right)^{n}\left(\operatorname{tang} \frac{1}{2} \theta\right)^{s}=\mu^{n-s}\left(1-\mu^{2}\right)^{\frac{1}{s}}\left(D_{\mu} \frac{1}{\mu}\right)^{n} \mu^{n+8}(1+\mu)^{n-s},
$$

où comme auparavant $\mu=\cos \theta$. En écrivant $\cot \theta=t$, et en faisant attention que le côté gauche de l'équation peut s'écrire sous la forme

$$
(\sin \theta)^{-n-1}\left(\sin ^{2} \theta D_{\theta}\right)^{n} \sin \theta\left(\operatorname{tang} \frac{1}{2} \theta\right)^{n},
$$

et que l'on a

$$
\sin \theta=\frac{1}{\sqrt{1+t^{2}}}, \quad \operatorname{tang} \frac{1}{2} \theta=\sqrt{1+t^{2}}-t, \quad \sin ^{2} \theta D_{\theta}=-D_{t},
$$

${ }^{1}$ Le lecteur pourrait omettre cet article, qui ne fait que montrer qu'une certaine formule du Professeur Donkin se réduit à son identité seconde.

C. IV. 
le côté gauche devient

$$
(-)^{n}\left(1+t^{2}\right)^{\frac{1}{2}(n+1)} D_{t}{ }^{n} \frac{\left(\sqrt{1+t^{2}}-t\right)^{s}}{\sqrt{1+t^{2}}} .
$$

Le côté droit peut s'écrire sous la forme

$$
\mu^{n-s+1}\left(1-\mu^{2}\right)^{\frac{1}{s}}\left(\frac{1}{\mu} D_{\mu}\right)^{n} \mu^{n+8-1}(1+\mu)^{n+s},
$$

et en observant que

$$
\mu=\frac{t}{\sqrt{1+t^{2}}}, \quad \frac{1}{\mu} D_{\mu}=\frac{\left(1+t^{2}\right)^{2}}{t} D_{t}
$$

le côté droit devient

$$
\frac{t^{n-s+1}}{\left(1+t^{2}\right)^{\frac{1}{2}(n+1)}}\left(\frac{\left(1+t^{2}\right)^{2}}{t} D_{t}\right)^{2} \frac{t^{n+s-1}}{\left(1+t^{2}\right)^{n-\frac{1}{2}}\left(\sqrt{ } 1+t^{2}-t\right)^{n-s}} ;
$$

en comparant les deux expressions on obtient

$$
(-)^{n} \frac{\left(1+t^{2}\right)^{n+1}}{t^{n-s+1}} D_{t}{ }^{n} \frac{\left(\sqrt{1+t^{2}}-t\right)^{s}}{\sqrt{1+t^{2}}}=\left(\frac{\left(1+t^{2}\right)^{2}}{t} D_{t}\right)^{n} \frac{t^{n+s-1}}{\left(1+t^{2}\right)^{n-\frac{1}{2}}\left(\sqrt{ } 1+t^{2}-t\right)^{n-s}},
$$

et de là, en écrivant comme auparavant $i=\sqrt{-1}$, et

$$
\sqrt{1+t^{2}}-t=-\frac{1}{2}(\sqrt{t+i}-\sqrt{t-i})^{2}
$$

la formule devient

$$
\left(\frac{\left(1+t^{2}\right)^{2}}{2 t} D_{t}\right)^{n} \frac{(2 t)^{n+8-1}}{\left(1+t^{2}\right)^{n-\frac{1}{2}}(\sqrt{1+i}-\sqrt{t-i})^{2 n-2 s}}=\frac{\left(1+t^{2}\right)^{n+1}}{(2 t)^{n-8+1}} D_{t}{ }^{n} \frac{(\sqrt{t+i}-\sqrt{t-i})^{2 s}}{\sqrt{1+t^{2}}},
$$

laquelle est un cas particulier de

$$
\left[\frac{[(x+\lambda)(x+\mu)]^{2}}{2 x+\lambda+\mu} D_{x}\right]^{n} \frac{(2 x+\lambda+\mu)^{n+8-1}}{[(x+\lambda)(x+\mu)]^{n-\frac{1}{2}}(\sqrt{x+\lambda}-\sqrt{ } x+\mu)^{2 n-2 s}}
$$

$$
=\frac{[(x+\lambda)(x+\mu)]^{n+1}}{(2 x+\lambda+\mu)^{n-8+1}} D_{x}{ }^{n} \frac{(\sqrt{x+\lambda}-\sqrt{x+\mu})^{2 s}}{\sqrt{(x+\lambda)(x+\mu})}
$$

c'est-à-dire de

$$
\left(P^{-1} Q^{4} D_{x}\right)^{n}\left(P^{n+8-1} Q^{-2 n+1} R^{-n+8}\right)=P^{-n+8-1} Q^{2 n+2} D_{x}^{n}\left(Q^{-1} R^{s}\right) .
$$

4. En écrivant comme auparavant

$$
P=2 x+\lambda+\mu, \quad Q=\sqrt{(x+\lambda)(x+\mu)}, \quad R=(\sqrt{x+\lambda})-\sqrt{x+\mu})^{2},
$$

on a $R=P-2 Q$, et en écrivant pour abréger $(\lambda-\mu)^{2}=\Lambda$, 
on obtient aussi

$$
\Lambda=P^{2}-4 Q^{2}, \quad \frac{\Lambda}{R}=P+2 Q
$$

on trouve de plus

$$
D_{x} P=2, \quad D_{x} Q=\frac{1}{2} \frac{P}{Q}, \quad D_{x} R=-\frac{R}{Q} .
$$

5. Les dernières formules donnent

$$
D_{x} P^{\alpha} Q^{\beta} R^{\gamma}=\frac{1}{2} \beta P^{\alpha+1} Q^{\beta-2} R^{\gamma}-\gamma P^{\alpha} Q^{\beta-1} R^{\gamma}+2 \alpha P^{\alpha-1} Q^{\beta} R^{\gamma},
$$

formule dont on pourrait se servir pour chercher $D_{x}^{r} P^{\alpha} Q^{\beta} R^{\gamma}$; on a par exemple

$$
\begin{aligned}
D_{x}^{2} P^{\alpha} Q^{\beta} R^{\gamma} & =\frac{1}{4} \beta(\beta-2) P^{a+2} Q^{\beta-4} R^{\gamma}-\frac{1}{2} \gamma(2 \beta-1) P^{a+1} Q^{\beta-3} R^{\gamma} \\
+ & {\left[\beta(2 \alpha+1)+\gamma^{2}\right] P^{\alpha} Q^{\beta-2} R^{\gamma}-4 \alpha \gamma P^{\alpha-1} Q^{\beta-1} R^{\gamma}+4(\alpha-1) \gamma P^{a-2} Q^{\beta} R^{\gamma}, }
\end{aligned}
$$

et ainsi de suite. Et de même

$$
P^{-1} Q^{4} D_{x} P^{\alpha} Q^{\beta} R^{\gamma}=\frac{1}{2} \beta P^{a} Q^{\beta+2} R^{\gamma}-\gamma P^{a-1} Q^{\beta+3} R^{\gamma}+2 \alpha P^{\alpha-2} Q^{\beta+4} R^{\gamma},
$$

et de là

$$
\begin{gathered}
\left(P^{-1} Q^{4} D_{x}\right)^{2} P^{a} Q^{\beta} R^{\gamma}=\frac{1}{4} \beta(\beta+2) P^{\alpha} Q^{\beta+4} R^{\gamma}-\frac{1}{2}(2 \beta+3) \gamma P^{a-1} Q^{\beta+5} R^{\gamma} \\
+\left[2 \alpha(\beta+2)+\gamma^{2}\right] P^{\alpha-2} Q^{\beta+6} R^{\gamma}-2(2 \alpha-1) \gamma P^{a-3} Q^{\beta+7} R^{\gamma} \\
+4 \alpha(\alpha-2) P^{\alpha-4} Q^{\beta+8} R^{\gamma}
\end{gathered}
$$

et ainsi de suite. Mais il serait difficile d'obtenir de cette manière l'expression de la $r$-ième répétition de l'opération $D_{x}$, ou $P^{-1} Q^{4} D_{x}$, sur $P^{a} Q^{\beta} R^{\gamma}$ et je ne poursuis pas la question.

6. Je vais à présent chercher le développement de $D_{x}^{r} P^{\alpha} Q^{\beta} R^{\gamma}(\alpha=0)$ ou ce qui est la même chose $D_{x}^{r} Q^{\beta} R^{\gamma}$. On a

$$
D_{x} Q^{\beta} R^{\gamma}=\frac{1}{2} \beta P Q^{\beta-2} R^{\gamma}-\gamma Q^{\beta-1} R^{\gamma},
$$

ou en substituant pour $P$ la valeur

$$
P=\frac{\Lambda}{R}-2 Q,
$$

on a

$$
D_{x} Q^{\beta} R^{\gamma}=-(\beta+\gamma) Q^{\beta-1} R^{\gamma}-\frac{1}{2} \beta \Lambda Q^{\beta-2} R^{\gamma-1} .
$$

La répétition de l'opération $D_{x}$ donne évidemment une expression de la forme

$$
\begin{aligned}
& D_{x}^{r} Q^{\beta} R^{\gamma}=(-)^{r} \quad L_{r} \quad \Lambda^{0} Q^{\beta-r} \quad R^{\gamma} \\
& \begin{aligned}
& \vdots \\
+(-)^{r-\theta} & \frac{1}{2^{\theta}} L_{r, \theta} \quad \Lambda^{\theta} Q^{\beta-r-\theta} R^{\gamma-\theta}
\end{aligned} \\
& +\quad \frac{1}{2^{r}} L_{r, \mu r} \cdot \Lambda^{r} Q^{\beta-2 r} R^{r-r},
\end{aligned}
$$


et on obtient pour $L_{r, \theta}$ l'équation aux différences

$$
L_{r+1, \theta+1}-(\beta+\gamma-r-2 \theta-2) L_{r, \theta+1}-(\beta-r-\theta) L_{r, \theta}=0,
$$

laquelle, avec les conditions particulières

$$
L_{r,-1}=0, \quad L_{r, r+1}=0, \quad L_{0,0}=1,
$$

suffit pour déterminer les coefficients $L_{r},{ }_{\theta}$ de la formule.

7. Avant d'aller plus loin, je vais considérer le cas particulier $\beta=0$. L'équation aux différences est ici

$$
L_{r+1, \theta+1}-(\gamma-r-2 \theta-2) L_{r, \theta+1}+(r+\theta) L_{r, \theta}=0,
$$

et l'on satisfait à cette équation en posant

$$
L_{r, \theta}=\frac{(-)^{\theta}[r+\theta-1]^{2 \theta}[\gamma-\theta-1]^{r-\theta-1}}{2^{\theta}[\theta]^{\theta}},
$$

ou, ce qui est la même chose,

$$
L_{r, \theta}=\frac{(-)^{\theta} 2^{\theta} \gamma\left[\theta-\frac{1}{2}\right]^{\theta}[r+\theta-1]^{r-\theta-1}[\gamma-\theta-1]^{r-\theta-1}}{[r-\theta-1]^{r-\theta-1}} .
$$

En effet on déduit de la première expression

$$
\begin{aligned}
L_{r+1, \theta+1}-(\gamma-r-2 \theta-2) L_{r, \theta+1} & \\
=\frac{(-)^{\theta+1} \gamma}{2^{\theta+1}[\theta+1]^{\theta+1}}(r & +\theta)[r+\theta-1]^{2 \theta}[\gamma-\theta-2]^{r-\theta-2} \\
\times & \times[(r+\theta+1)(\gamma-r)-(\gamma-r-2 \theta-2)(r-\theta-1)],
\end{aligned}
$$

et le facteur en [ ] est $2(\theta+1)(\gamma-\theta-1)$, de manière que l'expression devient

ce qui est

$$
=\frac{-(-)^{\theta} \gamma}{2^{\theta}[\theta]^{\theta}}(r+\theta)[r+\theta-1]^{2 \theta}[\gamma-\theta-1]^{r-\theta-1},
$$

$$
=-(r+\theta) L_{r, \theta} .
$$

L'équation aux différences est donc satisfaite; les conditions pour les limites sont aussi satisfaites, et la valeur qui vient d'être donnée est donc celle de $L_{r, \theta}$ dans le cas particulier dont il s'agit où $\beta=0$.

8. Il sera convenable d'écrire

$$
\frac{(-)^{\theta}}{2^{\theta} \gamma} L_{r, \theta}=\frac{[r+\theta-1]^{2 \theta}[\gamma-\theta-1]^{r-\theta-1}}{2^{2 \theta}[\theta]^{\theta}}=\frac{\left[\theta-\frac{1}{2}\right]^{\theta}[r+\theta-1]^{r-\theta-1}[\gamma-\theta-1]^{r-\theta-1}}{[r-\theta-1]^{r-\theta-1}}=L_{r, \theta}^{\prime},
$$

et alors en observant que $L_{r, \theta}$, ou $L_{r, \theta}^{\prime}$ se réduit à zéro pour $\theta=r$, on obtient

$$
\frac{(-)^{r}}{\gamma} D_{x}^{r} R^{\gamma}=L_{r, 0}^{\prime} Q^{-r} R^{\gamma} \ldots+L_{r, \theta}^{\prime} \Lambda^{\theta} Q^{-r-\theta} R^{\gamma-\theta} \ldots+L_{r, r-1}^{\prime} \Lambda^{r-1} Q^{-2 r+1} R^{\gamma-r-1} .
$$


9. Cela est en effet sous une forme un peu modifiée la formule fondamentale de mon mémoire dans le Camb. and Dub. Math. Journal; la valeur qu'on y trouve du coefficient $K_{r, \theta}$ (en mettant $\gamma$ au lieu de $i$ est)

$$
K_{r, \theta}=\frac{\Gamma\left(r-\frac{1}{2}-\theta\right) \Gamma(2 r-1-\theta) \Gamma(\gamma-r-\theta-1)}{\Gamma\left(\frac{1}{2}\right) \Gamma(\theta+1) \Gamma(2 r-1-2 \theta) \Gamma(\gamma-r+1)},
$$

et le coefficient $L_{r, \theta}(\beta=0)$ du présent mémoire est lié avec $K_{r, \theta}$ par l'équation

$$
\frac{(-)^{\theta}}{2^{\theta} \gamma} L_{r, \theta}=L_{r, \theta}^{\prime}=K_{r, r-1-\theta}
$$

La valeur de $L_{r, r-1}^{\prime}$ est $\left[r-\frac{3}{2}\right]^{r-1}$, et pour $r=\gamma+1$ le coefficient devient $\left[\gamma-\frac{1}{2}\right]^{\gamma}$, et tous les autres coefficients se réduisent à zéro: la formule devient donc tout simplement

$$
\frac{(-)^{\gamma+1}}{\gamma} D_{x^{\gamma+1}} R^{\gamma}=\left[\gamma-\frac{1}{2}\right]^{\gamma} \Lambda^{\gamma} Q^{-2 \gamma-1} \text {. }
$$

10. Dans le cas $r>\gamma+1$ il convient de modifier la forme de l'équation. J'écris $r=\gamma+1+s$, puis en faisant attention que les coefficients $L_{\gamma+1+s, \theta}^{\prime}$ se réduisent à zéro pour $\theta<\gamma$, on peut écrire $\gamma+\theta$ au lieu de $\theta$, et dans la formule nouvelle étendre $\theta$ depuis $\theta=0$ jusqu'à $\theta=s$. On a ainsi

$$
\begin{array}{rlll}
\frac{(-)^{\gamma+1+8}}{\gamma} D_{x^{\gamma+1+8}} R^{\gamma}= & L_{\gamma+1+\delta, \gamma}^{\prime} \quad \Lambda^{\gamma} Q^{-2 \gamma-1-8} & R^{0} \\
& \vdots \\
& +L_{\gamma+1+8, \gamma+\theta}^{\prime} \Lambda^{\gamma+\theta} Q^{-2 \gamma-1-8-\theta} & R^{-\theta} \\
& \vdots \\
& +L_{\gamma+1+\delta}^{\prime},_{\gamma+\delta} \Lambda^{\gamma+\delta} Q^{-2 \gamma-1-2 s} \quad R^{-8}
\end{array}
$$

et dans cette équation le côté gauche peut s'exprimer sous la forme

On obtient ainsi

$$
L_{\gamma+1, \gamma}^{\prime} \Lambda^{\gamma}(-)^{s} D_{x}^{s} Q^{-2 \gamma-1} \text {. }
$$

$$
\begin{aligned}
D_{x}^{s} Q^{-2 \gamma-1}= & L_{s, 0}^{\prime \prime} \quad Q^{-2 \gamma-1-s} \\
& \vdots \\
+ & L_{s, \theta}^{\prime \prime} \Lambda^{\theta} Q^{-2 \gamma-1-8-\theta} R^{-\theta} \\
& \vdots \\
& +L_{s, s}^{\prime \prime} \Lambda^{s} Q^{-2 \gamma-1-2 s} \quad R^{-s},
\end{aligned}
$$

où $L_{s, \theta}^{\prime \prime}=(-)^{s} L_{\gamma+1+s, \gamma+\theta}^{\prime}-L_{\gamma+1, \gamma}^{\prime}$, et en substituant pour les coefficients $L^{\prime}$ leurs valeurs, et en faisant attention que $[-\theta-1]^{s-\theta}=(-)^{s-\theta}[s]^{s-\theta}$,

on trouve

$$
L_{s, \theta}^{\prime \prime}=\frac{(-)^{\theta}[2 \gamma+s+\theta]^{2 \gamma+2 \theta}[s]^{s-\theta}}{2^{2 \theta}[2 \gamma]^{2 \theta}[\gamma+\theta]^{\theta}}=\frac{(-)^{\theta}\left[\gamma+\theta-\frac{1}{2}\right]^{\theta}[2 \gamma+s+\theta]^{s-\theta}[s]^{s-\theta}}{[s-\theta]^{s-\theta}}
$$

la formule qui vient d'être trouvée est donnée sous une forme différente dans mon mémoire déjà cité. 
11. Je résume l'équation générale

$$
L_{r+1, \theta+1}-(\beta+\gamma-r-2 \theta-2) L_{r, \theta+1}-(\beta-r-\theta) L_{r, \theta}=0,
$$

les conditions aux limites étant comme auparavant

On a en particulier

$$
L_{r,-1}=0, \quad L_{r, r+1}=0, \quad L_{0,0}=1 .
$$

$$
\begin{array}{llr}
L_{r+1,0}-(\beta+\gamma-r & ) L_{r, 0} & =0 \\
L_{r+1,1}-(\beta+\gamma-r-2) L_{r, 1}-(\beta-r & ) L_{r, 0}=0 \\
L_{r+1,2}-(\beta+\gamma-r-4) L_{r, 2}-(\beta-r-1) L_{r, 1} & =0 \\
\vdots & -(\beta-2 r & ) L_{r, r}=0
\end{array}
$$

De là on voit tout de suite que les valeurs de $L_{r},{ }_{0}, \mathrm{~L}_{r}, r$ sont

$$
L_{r,{ }_{0}}=[\beta+\gamma]^{r}, \quad L_{r}, r=[\beta,-2]^{r},
$$

où dans la dernière équation la notation au côté droit dénote une factorielle à différence -2 .

12. Les autres coefficients $L_{r, 1}, L_{r, 2}$, etc. peuvent s'obtenir successivement par une intégration directe des équations; et quoiqu'on ne les obtienne pas de cette manière sous la forme la plus commode, cependant il convient de donner l'investigation. Pour $L_{r, 1}$ on peut écrire

l'équation pour $M_{r, 1}$ sera

$$
L_{r, 1}=[\beta+\gamma-2]^{r} M_{r, 1},
$$

$$
M_{r+1,1}-M_{r, 1}=\frac{(\beta-r)[\beta+\gamma]^{r}}{[\beta+\gamma-2]^{r+1}},=\frac{(\beta-r)(\beta+\gamma)(\beta+\gamma-1)}{(\beta+\gamma-r)(\beta+\gamma-r-1)(\beta+\gamma-r-2)},
$$

laquelle peut aussi s'écrire

$$
M_{r+1,1}-M_{r, 1}=1+\frac{-\frac{1}{2}(r+2)(r+1)(\gamma-2)}{\beta+\gamma-r-2}+\frac{(r+1) r(\gamma-1)}{\beta+\gamma-r-1}+\frac{-\frac{1}{2} r(r-1) \gamma}{\beta+\gamma-r} .
$$

Cette équation a une solution

cela donne en effet

$$
M_{r, 1}=r+\frac{A_{r}}{\beta+\gamma-r-1}+\frac{B_{r}}{\beta+\gamma-r}
$$

$$
M_{r+1,1}-M_{r, 1}=1+\frac{A_{r+1}}{\beta+\gamma-r-2}+\frac{B_{r+1}-A_{r}}{\beta+\gamma-r-1}+\frac{-B_{r}}{\beta+\gamma-r},
$$

et l'on doit ainsi avoir

$$
A_{r+1}=-\frac{1}{2}(r+2)(r+1)(\gamma-2), \quad B_{r+1}-A_{r}=(r+1) r(\gamma-1), \quad-B_{r}=-\frac{1}{2} r(r-1) \gamma,
$$

équations qui sont satisfaites par

$$
A_{r}=-\frac{1}{2}(r+1) r(\gamma-2), \quad B_{r}=\frac{1}{2} r(r-1) \gamma
$$

Les conditions aux limites sont aussi satisfaites, et en formant l'expression de $L_{r+1}$ on obtient

$$
L_{r, 1}=[\beta+\gamma-2]^{r}\left(r+\frac{-\frac{1}{2}(r+1) r(\gamma-2)}{\beta+\gamma-r-1}+\frac{\frac{1}{2} r(r-1) \gamma}{\beta+\gamma-r}\right) .
$$


13. La valeur de $L_{r, 2}$ peut s'obtenir par un procédé semblable, et l'on a où

$$
L_{r, 2}=[\beta+\gamma-4]^{r}\left(\frac{1}{2} r(r-1)+\frac{A_{r}}{\beta+\gamma-r-3}+\frac{B_{r}}{\beta+\gamma-r-2}+\frac{C_{r}}{\beta+\gamma-r-1}+\frac{D_{r}}{\beta+\gamma-r}\right),
$$

$$
\begin{aligned}
& A_{r}=\frac{1}{48}(r+1) r\left(\left(r^{2}+5 r-6\right) \gamma^{2}+\left(-11 r^{2}-31 r+42\right) \gamma+\left(24 r^{2}+48 r-72\right)\right) \\
& B_{r}=-\frac{3}{48} r(r-1)\left(\left(r^{2}+3 r-2\right) \gamma^{2}+\left(-7 r^{2}-13 r+6\right) \gamma+8 r^{2}+16 r\right), \\
& C_{r}=\frac{3}{48}(r-1)(r-2)\left(\left(r^{2}+r\right) \gamma^{2}+\left(-3 r^{2}-3 r\right) \gamma\right), \\
& D_{r}=-\frac{1}{48}(r-2)(r-3)\left(\left(r^{2}-r\right) \gamma^{2}+\left(r^{2}-r\right) \gamma\right),
\end{aligned}
$$

et l'on peut remarquer que les suites des coefficients numériques $(1,1,1,1),(5,3,1,-1)$, $(-6,-2,0,0)$ ont respectivement les premières, secondes, et troisièmes différences égales à zéro, les suites $(-11,-7,-3,1),(-31,-13,-3,-1)$ ont respectivement les secondes, et troisièmes différences égales à zéro, et la suite $(24,8,0,0)$ a les troisièmes différences égales à zéro. Je remarque que pour $r=1, r=2$ les expressions de $L_{r, 1}, L_{r, 2}$ donnent

$$
\begin{aligned}
& L_{1,1}=(\beta+\gamma-2)\left(1-\frac{(\gamma-2)}{\beta+\gamma-2}\right),=\beta, \\
& L_{2,2}=(\beta+\gamma-4)(\beta+\gamma-5)\left(1+\frac{\gamma^{2}-8 \gamma+15}{\beta+\gamma-5}+\frac{-\gamma^{2}+6 \gamma-8}{\beta+\gamma-4}\right),=\beta(\beta-2),
\end{aligned}
$$

lesquelles s'accordent avec les résultats donnés par l'expression générale de $L_{r, r}$.

14. L'expression de $L_{r, 1}$ peut se transformer en

$$
L_{r, 1}=r \beta[\beta+\gamma-2]^{r-1}-\frac{1}{2} r(r-1)[\beta+\gamma-2]^{r-2},
$$

et l'expression de $L_{r, 2}$ peut de même, avec beaucoup plus de peine, se transformer en

$$
L_{r, 2}=\frac{1}{2} r(r-1)\left\{\begin{array}{l}
\beta(\beta-2)[\beta+\gamma-4]^{r-2} \\
-\quad(r-2) \beta(\beta-1)[\beta+\gamma-4]^{r-3} \\
+\frac{1}{2}(r-2)(r-3) \frac{1}{2}(\beta+1) \beta[\beta+\gamma-4]^{r-4}
\end{array}\right.
$$

et la forme de ces équations et d'autres considérations m'ont conduit à assumer en général

$$
\begin{aligned}
& L_{r, \theta}=\frac{1}{[\theta]^{\theta}} \quad A_{\theta, 0}[r]^{\theta} \quad[\beta+\gamma-2 \theta]^{r-\theta} \\
& -\frac{\vdots}{[\theta-1]^{\theta-1}[1]^{1}} \quad \frac{1}{2} A_{\theta, 1}[r]^{\theta+1}[\beta+\gamma-2 \theta]^{r-\theta-1} \\
& +\frac{(-)^{\sigma}}{[\theta-\sigma]^{\theta-\sigma}[\sigma]^{\sigma}} \cdot \frac{1}{2^{\sigma}} A_{\theta, \sigma}[r]^{\theta+\sigma}[\beta+\gamma-2 \theta]^{\gamma-\theta-\sigma} \\
& \frac{(-)^{\theta}}{[\theta]^{\theta}} \quad A_{\theta, \theta}[r]^{2 \theta} \quad[\beta+\gamma-2 \theta]^{r-2 \theta} .
\end{aligned}
$$


15. En posant pour plus de commodité $\theta-1$ au lieu de $\theta$ dans l'équation aux différences, cette équation devient

$$
L_{r+1, \theta}-(\beta+\gamma-r-2 \theta) L_{r, \theta}=(\beta-r-\theta+1) L_{r, \theta-1},
$$

et puis substituant dans cette équation la valeur assumée de $L_{r, \theta}$, et les valeurs correspondantes de $L_{r+1, \theta}$ et $L_{r, \theta-1}$, le terme général au côté gauche sera

$$
\begin{aligned}
\frac{(-)^{\sigma}}{[\sigma]^{\sigma}[\theta-\sigma]^{-\sigma}} & \frac{1}{2^{\sigma}}[r]^{\theta+\sigma-1}[\beta+\gamma-2 \theta]^{r-\theta-\sigma} A_{\theta, \sigma} \\
\times & {[(r+1)(\beta+\gamma-r-\theta-\sigma)-(\beta+\gamma-r-2 \theta)(r-\theta-\sigma+1)] }
\end{aligned}
$$

l'expression en [ ] est $(\theta+\sigma)(\beta+\gamma-2 \theta+1)$, et en substituant cette valeur le terme général au côté gauche devient

$$
\frac{(-)^{\sigma}}{[\sigma]^{\sigma}[\theta-\sigma]^{\theta-\sigma}} \cdot \frac{1}{2^{\sigma}}(\theta+\sigma)[r]^{\theta+\sigma-1}[\beta+\gamma-2 \theta+1]^{r+1-\theta-\sigma} A_{\theta, \sigma} .
$$

Le terme général au côté droit est

$$
\begin{aligned}
\frac{(-)^{\sigma}}{[\sigma]^{\sigma}[\theta-1-\sigma]^{\theta-1-\sigma}} & \frac{1}{2^{\sigma}}[r]^{\theta-1-\sigma}[\beta+\gamma-2 \theta+1]^{r-\theta-\sigma} A_{\theta-1, \sigma} \\
& \times(\beta-\gamma-\theta+1)(\beta+\gamma-2 \theta+2),
\end{aligned}
$$

dont le dernier facteur est égal à

$$
(\beta+\gamma-r-\theta+1+\sigma)(\beta-2 \theta+2-\sigma)-(r-\theta+1-\sigma)(\gamma+\sigma)
$$

et en substituant cette valeur le terme devient

$$
\begin{aligned}
& \frac{(-)^{\sigma}}{[\sigma]^{\sigma}[\theta-1-\sigma]^{\theta-1-\sigma}} \frac{1}{2^{\sigma}}[r]^{\theta-1+\sigma}[\beta+\gamma-2 \theta-1]^{r-\theta-\sigma+1}(\beta-2 \theta+2-\sigma) A_{\theta-1, \sigma} \\
& -\frac{(-)^{\sigma}}{[\sigma]^{\sigma}[\theta-1-\sigma]^{\theta-1-\sigma}} \cdot \frac{1}{2^{\sigma}}[r]^{\theta+\sigma}[\beta+\gamma-2 \theta+1]^{r-\theta-\sigma}(\gamma+\sigma) A_{\theta-1, \sigma} .
\end{aligned}
$$

En écrivant dans la seconde ligne (ce qui est permis) $\sigma-1$ au lieu de $\sigma$, cette ligne devient

$$
-\frac{(-)^{\sigma-1}}{[\sigma-1]^{\sigma-1}[\theta-\sigma]^{\theta-\sigma}} \cdot \frac{1}{2^{\sigma-1}}[r]^{\theta+\sigma-1}[\beta+\gamma-2 \theta+1]^{r-\theta-\sigma+1}(\gamma+\sigma-1) A_{\theta-1, \sigma-1},
$$

et les deux lignes ensemble seront

$$
\begin{aligned}
\frac{(-)^{\sigma}}{[\sigma]^{\sigma}[\theta-\sigma]^{\theta-\sigma}} & \frac{1}{2^{\sigma}}[\gamma]^{\theta+\sigma-1}[\beta+\gamma-2 \theta+1]^{r-\theta-\sigma+1} \\
& \times\left[(\theta-\sigma)(\beta-2 \theta+2-\sigma) A_{\theta-1, \sigma}+2 \sigma(\gamma+\sigma-1) A_{\theta-1, \sigma-1}\right]
\end{aligned}
$$


ce qui est le terme général au côté droit. En comparant cela avec l'expression cidessus trouvée pour le terme général au côté gauche on obtient

$$
(\theta+\sigma) A_{\theta, \sigma}=(\theta-\sigma)(\beta-2 \theta+2-\sigma) A_{\theta-1, \sigma}+2 \sigma(\gamma+\sigma-1) A_{\theta-1, \sigma-1},
$$

pour l'équation aux différences à laquelle doit satisfaire le coefficient $A_{\theta, \sigma}$; en y écrivant

on aura pour $B_{\theta, \sigma}$ l'équation

$$
A_{\theta, \sigma}=[\gamma+\sigma-1]^{\sigma} B_{\theta, \sigma},
$$

$$
(\theta+\sigma) B_{\theta, \sigma}=(\theta-\sigma)(\beta-2 \theta+2-\sigma) B_{\theta-1, \sigma}+2 \sigma B_{\theta-1, \sigma-1},
$$

et on voit sans peine que les conditions aux limites sont

$$
B_{\theta,-1}=0, \quad B_{\theta, \theta+1}=0, \quad B_{0,0}=1 .
$$

16. On a en particulier

et de là

$$
B_{\theta, 0}=(\beta-2 \theta+2) B_{\theta-1,0}, \quad B_{\theta, \theta}=B_{\theta-1, \theta-1},
$$

$$
B_{\theta, 0}=[\beta,-2]^{\theta}, \quad B_{\theta, \theta}=1 .
$$

Les autres fonctions $B_{\theta, \sigma}$ sont des fonctions rationnelles et entières de $\beta$, du degré $\theta-\sigma$, données par l'équation aux différences; mais les coefficients numériques des différentes puissances de $\beta$ n'admettent, à ce qu'il paraît, aucune expression simple; les fonctions $B_{\theta, \sigma}$ sont, pour ainsi dire, une espèce de transcendantes rationnelles propres pour exprimer la valeur de $L_{r, \theta}$, et il sera suffisant de tabuler les valeurs de $B_{\theta, \sigma}$ sans pousser plus loin la recherche de la loi de ces valeurs. On a en effet pour $B_{0,0}, B_{1,0}$, etc.... $B_{1,1}$ les valeurs

$$
\begin{array}{rrrl}
1, \beta, & \beta(\beta-2), & \beta(\beta-2)(\beta-4), & \beta(\beta-2)(\beta-4)(\beta-6), \text { etc. } \ldots \\
1, & \beta-1, & \beta^{2}-4 \beta+\frac{5}{2}, & \beta^{3}-9 \beta^{2}+\frac{43}{2} \beta-\frac{21}{2}, \\
1, & \beta-2, & \beta^{2}-6 \beta+7, \\
1, & \beta-3,
\end{array}
$$

17. Puis en substituant pour $A_{\theta, \sigma}$ la valeur $[\gamma+\sigma-1]^{\sigma} B_{\theta, \sigma}$ on trouve

$$
\begin{aligned}
& L_{r, \theta}=\frac{1}{[\theta]^{\theta}} \quad B_{\theta, 0} \quad[r]^{\theta} \quad[\beta+\gamma-2 \theta]^{r-\theta} \\
& +\frac{(-)}{[\theta-1]^{\theta-1}[1]^{1}} \quad \frac{1}{2} B_{\theta, 1}[\gamma]^{1}[r]^{\theta+1} \quad[\beta+\gamma-2 \theta]^{r-\theta-1} \\
& +\frac{(-)^{\sigma}}{[\theta-\sigma]^{\theta-\sigma}[\sigma]^{\sigma}} \frac{1}{2^{\sigma}} B_{\theta, \sigma}[\gamma+\sigma-1]^{\sigma}[\gamma]^{\theta+\sigma}[\beta+\gamma-2 \theta]^{r-\theta-\sigma} \\
& +\frac{(-)^{\theta}}{[\theta]^{\theta}} \quad \frac{1}{2^{\theta}} B_{\theta, \theta}[\gamma+\theta-1]^{\theta}[r]^{2 \theta}[\beta+\gamma-2 \theta]^{r-2 \theta},
\end{aligned}
$$

C. IV. 

๘

ce qui est l'expression de $L_{r, \theta}$ dans la formule

$$
\begin{aligned}
D_{x}^{r} Q^{\beta} B^{\gamma}= & (-)^{-r} \quad L_{r, \theta} \quad Q^{\beta-\gamma} \quad R^{\gamma} \\
& \vdots \\
& +(-)^{r-\theta} \frac{1}{2^{\theta}} L_{r, \theta} \Lambda^{\theta} Q^{\beta-r-\theta} R^{\gamma-\theta} \\
& \vdots \\
& +\quad \frac{1}{2^{r}} L_{r, r} \Lambda^{r} Q^{\beta-2 r} R^{\gamma-r} .
\end{aligned}
$$

$\S$ III.

18. Je passe à présent au développement de

$$
\left(P^{-1} Q^{4} D_{x}\right)^{r} P^{\alpha} \quad Q^{\beta} R^{\gamma}, \quad(\alpha=\gamma-\beta),
$$

ou, ce qui est la même chose,

$$
\left(P^{-1} Q^{4} D_{x}\right)^{r} P^{\gamma-\beta} Q^{\beta} R^{\gamma} .
$$

On a comme auparavant

$$
P^{-1} Q^{4} D_{x} P^{a} Q^{\beta} R^{\gamma}=\frac{1}{2} \beta P^{a} Q^{\beta+2} R^{\gamma}-\gamma P^{a-1} Q^{\beta+3} R^{\gamma}+2 \alpha P^{\alpha-2} Q^{\beta+4} R^{\gamma},
$$

et. de là

$$
\begin{array}{r}
P^{-1} Q^{4} D_{x} P^{\gamma-\beta} Q^{\beta} R^{\gamma}=\frac{1}{2} \beta P^{\gamma-\beta} Q^{\beta+2} R^{\gamma}-\gamma P^{\gamma-\beta-1} Q^{\beta+3} R^{\gamma}+2(\gamma-\beta) P^{\gamma-\beta-2} Q^{\beta+4} R^{\gamma}, \\
=\frac{1}{2} \beta(P-2 Q) P^{\gamma-\beta-1} Q^{\beta+2} R^{\gamma}-(\gamma-\beta)(P-2 Q) P^{\gamma-\beta-2} Q^{\beta+3} R^{\gamma},
\end{array}
$$

c'est-à-dire

$$
P^{-1} Q^{4} D_{x} P^{\gamma-\beta} Q^{\beta} R^{\gamma}=\frac{1}{2} \beta P^{\gamma-\beta-1} Q^{\beta+2} R^{\gamma+1}-(\gamma-\beta) P^{\gamma-\beta-2} Q^{\beta+3} R^{\gamma+1} .
$$

On peut donc écrire

$$
\begin{aligned}
\left(P^{-1} Q^{4} D_{x}\right)^{r} P^{\gamma-\beta} Q^{\beta} R^{\gamma=} & \frac{1}{2^{r}} N_{r, 0} P^{\gamma-\beta-r} Q^{\beta+2 r} R^{\gamma+r} \\
& \vdots \\
+ & \frac{(-)^{\theta}}{2^{r-\theta}} N_{r, \theta} P^{\gamma-\beta-r-\theta} Q^{\beta+2 r+\theta} R^{\beta+r} \\
& \vdots \\
+ & (-)^{r} N_{r, r} P^{\gamma-\beta-2 r} Q^{\beta+2 r} R^{\gamma+r}
\end{aligned}
$$

et on trouve pour $N_{r, \theta}$ l'équation aux différences

$$
N_{r+1, \theta+1}-(\beta+2 r+\theta+1) N_{r, \theta+1}-(\gamma-\beta-r-\theta) N_{r, \theta}=0,
$$

laquelle avec les conditions aux limites

$$
N_{r,-1}=0, \quad N_{r, r+1}=0, \quad N_{0,0}=1,
$$

détermine le coefficient $N_{r, \theta}$. 
On a, en particulier

$$
\begin{array}{lll}
N_{r+1,0}=(\beta+2 r) & N_{r, 0}, \\
N_{r+1,1}=(\beta+2 r+1) & N_{r, 1}+(\gamma-\beta-r) & N_{r, 0}, \\
N_{r+1,2}=(\beta+2 r+2) & N_{r, 2}+(\gamma-\beta-r-1) N_{r, 1}, \\
\vdots & \\
N_{r+1, r+1}=(\gamma-\beta-2 r) & N_{r, r},
\end{array}
$$

lesquelles donnent tout de suite

$$
N_{r, 0}=[\beta+2 r-2,-2]^{r}, \quad N_{r, r}=[\gamma-\beta,-2]^{r} .
$$

19. L'équation ressemble à celle pour $L_{r, \theta}$, et l'on pourrait croire que l'intégration s'accomplirait d'une manière semblable, mais cela n'est pas ainsi; car en considérant la seconde équation de la suite, c'est-à-dire

en $\mathrm{y}$ mettant

$$
N_{r+1,1}-(\beta+2 r+1) N_{r, 1}=(\gamma-\beta-r)[\beta+2 r-2,-2]^{r},
$$

on obtient

$$
N_{r, 1}=[\beta+2 r-1,-2]^{r} M_{r, 1}
$$

et de là

$$
[\beta+2 r+1,-2]^{r+1}\left(M_{r+1,1}-M_{r, 1}\right)=(\gamma-\beta-r)[\beta+2 r-2,-2]^{r},
$$

$$
M_{r+1,1}-M_{r, 1}=\frac{(\gamma-\beta-r)[\beta+2 r-2,-2]^{r}}{[\beta+2 r+1,-2]^{r+1}} ;
$$

mais les facteurs du numérateur, et du dénominateur ne sont pas ici (ce qui arrivait dans l'équation pour la quantité dénotée auparavant par le même symbole $M_{r, 1}$ ) de la même forme, et il n'y a pas de simplification dans la forme de la fraction. En écrivant successivement $r-1, r-2, \ldots 2,1,0$ pour $r$ on trouve

$$
\begin{aligned}
M_{r, 1}=\gamma-\beta+\frac{(\gamma-\beta-1) \beta}{\beta+3}+ & \frac{(\gamma-\beta-2)(\beta+2) \beta}{(\beta+5)(\beta+3)} \\
& \ldots+\frac{(\gamma-\beta-r+1)(\beta+2 r-4) \ldots(\beta+2) \beta}{(\beta+2 r-1) \ldots(\beta+5)(\beta+3)},
\end{aligned}
$$

et de là

$$
\begin{array}{r}
N_{r, 1}=(\beta+2 r-1) \ldots(\beta+5)(\beta+3)\left(\gamma-\beta+\frac{(\gamma-\beta-1) \beta}{\beta+3}+\frac{(\gamma-\beta-2)(\beta+2) \beta}{(\beta+5)(\beta+3)}\right. \\
\left.\ldots+\frac{(\gamma-\beta-r+1)(\beta+2 r-4) \ldots(\beta+2) \beta}{(\beta+2 r-1) \ldots(\beta+5)(\beta+3)}\right) .
\end{array}
$$

Il ne paraît pas que la suite en [ ] puissse être additionnée, et cela m'empêche de pousser plus loin la recherche de la forme des coefficients $N_{r, \theta}$; la solution, telle que je l'ai trouvée, est donc donnée par l'expression de $\left(P^{-1} Q^{4} D_{x}\right)^{r} P^{\gamma-\beta} Q^{\beta} R^{\gamma}$ en termes des coefficients numériques $N_{r, \theta}$, et par l'équation aux différences et conditions aux limites qui déterminent les coefficients $N_{r, \theta}$. 
$\S I V$.

20. La première des identités du Prof. Donkin est

$$
D_{x}^{n} Q^{-1} R^{n}=(-)^{n} \Lambda^{n}\left[n-\frac{1}{2}\right]^{n} Q^{-2 n-1} ;
$$

mais par une forme ci-dessus trouvée No. 9 en y écrivant $n$ au lieu de $\gamma$ on a

$$
\frac{(-)^{n+1}}{n} D_{x}^{n+1} \quad R^{n}=\Lambda^{n}\left[n_{\bullet}^{-}-\frac{1}{2}\right]^{n} Q^{-2 n-1},
$$

et, en remarquant que $D_{x} R^{n}=-n Q^{-1} R^{n}$, on voit que les deux formules sont identiques, et la vérité du théorème est ainsi démontrée.

21. La seconde des deux identités est

$$
P^{-n+8-1} Q^{2 n+2} D_{x}^{n} Q^{-1} R^{s}=\left(P^{-1} Q^{4} D_{x}\right)^{n} P^{n+s-1} Q^{-2 n+1} R^{-n+8} .
$$

On a par la formule (No. 10) en y mettant $s$ au lieu de $\gamma$ et $n+1$ au lieu de $r$,

où

$$
\begin{aligned}
\frac{(-)^{n+1}}{s} D_{x^{n+1}} R^{s}= & L_{n+1,0}^{\prime} \quad Q^{-n-1} \quad R^{s} \\
& \vdots \\
& +L_{n+1, \theta}^{\prime} \Lambda^{\theta} \quad Q^{-n-1-\theta} R^{s-\theta} \\
& \vdots \\
& +L_{n+1, n}^{\prime} \Lambda^{n} Q^{-2 n-1} \quad R^{s-n}
\end{aligned}
$$

$$
L_{n+1, \theta}^{\prime}=\frac{\left[\theta-\frac{1}{2}\right]^{\theta}[n+\theta]^{n-\theta}[s-\theta-1]^{n-\theta}}{[n-\theta]^{n-\theta}} .
$$

22. En renversant l'ordre des termes et mettant aussi $L_{n+1, \theta}^{\prime}=V_{n-\theta}$, de manière que

on obtient

$$
V_{\theta}=\frac{\left[n-\frac{1}{2}-\theta\right]^{n-\theta}[2 n-\theta]^{\theta}[s-n+\theta-1]^{\theta}}{\lfloor\theta]^{\theta}},
$$

$$
\begin{aligned}
& \frac{(-)^{n+1}}{s} D_{x^{n+1}} R^{s}=V_{0} \Lambda^{n} \quad Q^{-2 n-1} \quad R^{s-n} \\
& +\dot{V}^{\theta} \Lambda^{n-\theta} Q^{-2 n-1+\theta} R^{s-n+\theta} \\
& +V_{n} \quad Q^{-n-1} \quad R^{s},
\end{aligned}
$$

et en remarquant que $D_{x} R^{s}=-s Q^{-1} R^{s}$, et que l'on a aussi $\Lambda=P^{2}-4 Q^{2}$ et $R=P-2 Q$, de manière que $\Lambda R^{-1}=(P+2 Q)$, l'équation peut s'écrire sous la forme

$$
\begin{array}{ccc}
Q^{2 n+2} P^{-n+8-1} D_{x}^{n} Q^{-1} R=(-)^{n} \times & V_{0}(P+2 Q)^{n} & P^{-n+8-1} Q R^{s} \\
\vdots & & \\
+ & V_{\theta}(P+2 Q)^{n-\theta} & P^{-n+8-1} Q^{1+\theta} R^{s} \\
\vdots & \\
+V_{n} & P^{-n+8-1} Q^{1+n} R^{s}
\end{array}
$$


et en développant les binomiels on obtient pour $Q^{2 n+2} P^{-n+8-1} D_{x}{ }^{n} Q^{-1} R^{s}$ la valeur $(-)^{n} \times$ $\left(V_{0}\right) P^{s-1} Q R^{s}+\left(\frac{[n]^{1}}{[1]^{1}} 2^{1} V_{0}+V_{1}\right) P^{s-2} Q^{2} R^{s}+\left(\frac{[n]^{2}}{[2]^{2}} 2^{2} V_{0}+\frac{[n-1]^{1}}{[1]^{1}} 2^{1} V_{1}+V_{2}\right) P^{s-3} Q^{3} R^{s}$

Cela devrait être égal à

$$
\cdots+\left(2^{n} V_{0}+2^{n-1} V_{1} \ldots+2^{1} V_{n-1}+V_{n}\right) P^{s-n-1} Q^{n+1} R^{s} \text {. }
$$

$$
\left(P^{-1} Q^{4} D_{x}\right)^{n} P^{n+8-1} Q^{-2 n+1} R^{-n+8}
$$

et le développement de cette dernière expression se déduit de celui (No. 18) de

$$
\left(P^{-1} Q^{4} D_{x}\right)^{r} P^{\gamma-\beta} \quad Q^{\gamma} \quad R^{\beta}
$$

en y écrivant $n,-2 n+1,-n+s$ au lieu de $r, \beta, \gamma$; la valeur sera ainsi

$$
=\frac{1}{2^{n}} N_{n, 0} P^{s-1} Q R^{s}-\frac{1}{2^{n-1}} N_{n, 1} P^{s-2} Q^{2} R^{s}+\frac{1}{2^{n-2}} N_{n, 2} P^{s-3} Q^{3} R^{s} \ldots+(-)^{n} N_{n, n} P^{s-n-1} Q^{n+1} R^{s},
$$

laquelle a la même forme que le développement de la fonction au côté gauche de l'équation, et l'identité des deux expressions dépend des équations

$$
\begin{gathered}
\frac{1}{2^{n}} N_{n, 0}=(-)^{n} \quad V_{0}, \\
\frac{1}{2^{n-1}} N_{n, 1}=(-)^{n-1}\left(\frac{[n]^{1}}{[1]^{1}} 2^{1} V_{0}+V_{1}\right), \\
\frac{1}{2^{n-2}} N_{n, 2}=(-)^{n-2}\left(\frac{[n]^{2}}{[2]^{2}} 2^{2} V_{0}+\frac{[n-1]^{1}}{[1]^{1}} 2^{1} V_{1}+V_{2}\right), \\
\vdots \\
N_{n, n}=\left(2^{n} V_{0}+2^{n-1} V_{1} \ldots+2 V_{n-1}+V_{n}\right) ;
\end{gathered}
$$

mais comme on ne sait pas la forme générale des coefficients $N_{n, \theta}$ je n'ai pas pu vérifier complètement ces équations. On a cependant en mettant $n,-2 n+1,-n+s$ pour $r, \beta, \gamma$

$$
\begin{aligned}
& N_{n, 0}=[-1,+2]^{n},=(-)^{n} \quad[2 n-1,-2]^{n},=(-1)^{n} 2^{n} \quad\left[n-\frac{1}{2}\right]^{n}, \\
& N_{n, 1}=s[-3,+2]^{n-1},=(-)^{n-1} s[2 n-1,-2]^{n-1},=(-)^{n-1} 2^{n-1} s\left[n-\frac{1}{2}\right]^{n-1},
\end{aligned}
$$

et les deux premières équations seront

$$
\left[n-\frac{1}{2}\right]^{n}=\left[n-\frac{1}{2}\right]^{n} ; \quad s\left[n-\frac{1}{2}\right]^{n-1}=\frac{n}{1} 2\left[n-\frac{1}{2}\right]^{n}+\left[n-\frac{3}{2}\right]^{n-1}(2 n-1)(s-n),
$$

où dans la seconde équation l'expression au côté droit est

$$
[2 n+2(s-n)]\left[n-\frac{1}{2}\right]^{n}=2 s\left[n-\frac{1}{2}\right]^{n}=s\left[n-\frac{1}{2}\right]^{n-1},
$$

comme cela devrait être. La dernière équation de la suite est

$$
N_{n, n}=2^{n} V_{0}+2^{n-1} V_{1} \ldots+2 V_{n-1}+V_{n}
$$

et comme on a $N_{n, n}=[n+s-1,-2]^{n}$, cette dernière équation peut aussi se vérifier. 